\title{
Diagnostic Efficacy of Serum Mac-2 Binding Protein Glycosylation Isomer and Other Markers for Liver Fibrosis in Non-Alcoholic Fatty Liver Diseases
}

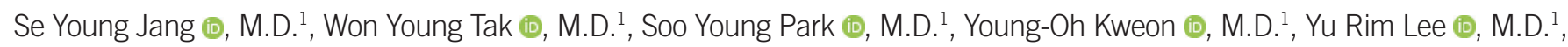

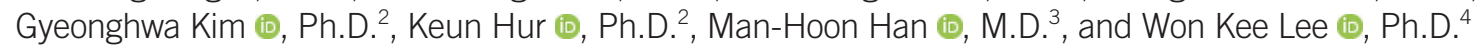

${ }^{1}$ Department of Internal Medicine, School of Medicine, Kyungpook National University, Kyungpook National University Hospital, Daegu, Korea; ${ }^{2}$ Department of Biochemistry and Cell Biology, Cell and Matrix Research Institute, School of Medicine, Kyungpook National University, Daegu, Korea; ${ }^{3}$ Department of Pathology, School of Medicine, Kyungpook National University, Kyungpook National University Hospital, Daegu, Korea; ${ }^{4}$ Department of Medical Informatics, School of Medicine, Kyungpook National University, Daegu, Korea

Background: Mac-2 binding protein glycosylation isomer (M2BPGi) has been established as a non-invasive biomarker for liver fibrosis. We evaluated the diagnostic efficacy of M2BPGi compared with those of other liver fibrosis markers in liver fibrosis in non-alcoholic fatty liver disease (NAFLD).

Methods: We analyzed serum M2BPGi levels in 113 NAFLD patients. A pathologist graded liver fibrosis histopathologically. The diagnostic efficacies of serum M2BPGi and other liver fibrosis markers (aspartate aminotransferase to platelet ratio index, fibrosis index based on four factors, and NAFLD fibrosis score [NFS]) were evaluated using correlation, area under the ROC curve (AUC), logistic regression, and C-statistics.

Results: Serum M2BPGi level and other liver fibrosis markers showed a moderate correlation with fibrosis grade. The AUC values of M2BPGi were $0.761,0.819,0.866$, and 0.900 for diagnosing fibrosis $(F)>0, F>1, F>2$, and $F>3$, respectively. Logistic regression analysis showed M2BPGi as the only independent factor associated with $\mathrm{F}>2$ and $\mathrm{F}>3$. Although C-statistics showed that NFS was the best diagnostic factor for F>2 and F>3, M2BPGi with NFS had an increased C-statistics value, indicating that it is a better diagnostic model.

Conclusions: The serum M2BPGi level increased with liver fibrosis severity and could be a good biomarker for diagnosing advanced fibrosis and cirrhosis in NAFLD patients. A well-controlled, prospective study with a larger sample size is needed to validate the diagnostic power of M2BPGi and other fibrosis markers in NAFLD.

Key Words: Mac-2 binding protein glycosylation isomer, Non-alcoholic fatty liver disease, Liver fibrosis, Diagnosis, Biomarker
Received: June 2, 2020

Revision received: July 23, 2020

Accepted: November 30, 2020

\section{Corresponding author:}

Won Young Tak, M.D., Ph.D.

Department of Internal Medicine, School of Medicine, Kyungpook National University, Kyungpook National University Hospital, 130 Dongdeok-ro, Jung-gu, Daegu 41944, Korea

Tel: +82-53-200-5519

Fax: +82-53-426-2046

E-mail: wytak@knu.ac.kr

\section{INTRODUCTION}

Serum Mac-2 binding protein glycosylation isomer (M2BPGi) is a novel glyco-biomarker of liver fibrosis. Wisteria floribunda agglutinin-positive Mac-2 binding protein (M2BP) is converted into
M2BPGi under liver fibrosis conditions. M2BPGi formation indicates changes in the sugar chain structure of M2BP and is correlated with the onset of liver fibrosis. M2BPGi has also been used as a serum biomarker for predicting the development of hepatocellular carcinoma (HCC) in patients with chronic hepati- 
tis B [1, 2], chronic hepatitis C [3], or non-alcoholic fatty liver disease (NAFLD) [4]. Moreover, it can be used as a serum biomarker for diagnosing liver fibrosis in patients with viral hepatitis, autoimmune hepatitis [5], primary biliary cholangitis [6, 7], or NAFLD $[8,9]$. The studies reporting these findings mainly involved Japanese patients; therefore, the applicability of serum M2BPGi level as a biomarker was recently validated in the Korean population [10-13].

NAFLD is an emerging condition worldwide. In recent years, NAFLD has become a highly prevalent form of liver disease and is a significant risk factor for the development of HCC in most developed countries [14-16]. NAFLD can progress to liver cirrhosis owing to sustained liver injury and restoration, which lead to distortion of the hepatic architecture and progressive hepatic fibrosis. Liver cirrhosis increases the risk of developing HCC, impairs the immune system by reducing complement levels, and deteriorates the quality of life in cases involving ascites, variceal bleeding, or encephalopathy. Therefore, evaluating the extent and degree of liver fibrosis is essential for predicting the clinical outcomes of patients with NAFLD. Liver biopsy is the gold standard for evaluating liver fibrosis. However, liver biopsy is invasive, expensive, and susceptible to sampling errors, and it does not reflect the state of the whole liver [17]. Currently, transient elastography (TE; FibroScan, Echosens, Paris, France) and magnetic resonance elastography (MRE) are used to noninvasively evaluate liver fibrosis in patients with NAFLD. Other measures used in clinical practice include scoring models, such as aspartate aminotransferase (AST) to platelet ratio index (APRI), fibrosis index based on four factors (FIB-4), and NAFLD fibrosis score (NFS). We analyzed the diagnostic efficacy of serum M2BPGi compared with other markers in liver fibrosis in NAFLD.

\section{MATERIALS AND METHODS}

\section{Patients}

We included 113 patients who had undergone liver biopsy and were diagnosed as having NAFLD between March 2015 and March 2018 at Kyungpook National University Hospital, Daegu, Korea in this retrospective study. We excluded patients who consumed significant amounts of alcohol or had coexisting chronic liver diseases caused by hepatitis viruses. Routine biochemistry analyses, including liver function test and analyses of the lipid profile and complete blood count, were performed within a week before liver biopsy. This study was conducted according to local ethical guidelines, in accordance with the Declaration of Helsinki, 2013, and was approved by the Institutional Review Board of
Kyungpook National University Hospital (KNUH-2017-10-031). All patients provided informed consent prior to liver biopsy and sample collection.

\section{Histological evaluation}

Each NAFLD patient underwent ultrasonography-guided liver biopsy for diagnosis. Biopsied liver samples were fixed in formalin and embedded in paraffin. Diagnosis required a minimum of six portal tracts in the samples. Slides were stained with hematoxylin and eosin and Masson's trichrome. All liver samples were evaluated by a single, experienced liver pathologist who was double-blinded to the clinical data. Liver fibrosis stage (F) was assessed according to the system devised by the Pathology Committee of the Nonalcoholic Steatohepatitis (NASH) Clinical Research Network [18]; the NAFLD activity score (NAS) represents the sum of the steatosis (0-3), lobular inflammation (0-3), and ballooning (0-2) scores. Significant and advanced fibrosis were defined as $F>1$ and $F>2$, respectively [9].

\section{Measurement of serum M2BPGi level}

Peripheral blood was collected during liver biopsy. Serum was separated by centrifuging $5 \mathrm{~mL}$ of blood at $1,800 \times \mathrm{g}$ for $10 \mathrm{~min}$ utes at $4^{\circ} \mathrm{C}$ and then stored at $-80^{\circ} \mathrm{C}$ for $3-34$ months. The serum M2BPGi level was measured using a Wisteria floribunda agglutinin antibody immunoassay, with a chemiluminescence enzyme immunoassay system (HISCL-2000i; Sysmex, Kobe, Japan), as previously reported [19, 20]. The measured serum M2BPGi levels were used to calculate the M2BPGi level cut-off index (COI) using the following equation:

$$
\begin{aligned}
& \text { cut-off index }(\mathrm{COI})=\left([\mathrm{M} 2 \mathrm{BPGi}]_{\text {sample }}-[\mathrm{M} 2 \mathrm{BPGi}]_{\text {negative control }}\right) / \\
& \left([\mathrm{M} 2 \mathrm{BPGi}]_{\text {positive control- }}-[\mathrm{M} 2 \mathrm{BPGi}]_{\text {negative control }}[9]\right. \text {. }
\end{aligned}
$$

\section{Clinical and laboratory data}

Clinical data, including sex, age, weight, height, and body mass index (BMI), were collected from each patient. BMI was calculated as weight $(\mathrm{kg})$ divided by height $(\mathrm{m})$ squared. Additionally, platelet count, AST, alanine aminotransferase (ALT), gamma-glutamyl transferase (GGT), albumin, cholesterol, and fasting plasma glucose levels were measured. Three liver fibrosis scores employing the serum M2BPGi level for liver fibrosis confirmation were compared: APRI [21]: (AST (U/L)/upper normal limit× 100)/ platelet count $\left(\times 10^{9} / \mathrm{L}\right)$; FIB-4 [22]: age (year) $\times$ AST $(\mathrm{U} / \mathrm{L}) /$ platelet count $\left(\times 10^{9} / \mathrm{L}\right) \times \sqrt{ }$ ALT $(\mathrm{U} / \mathrm{L})$; and NFS [23]: $-1.675+0.037$ $\times$ age (year) $+0.094 \times \mathrm{BMI}\left(\mathrm{kg} / \mathrm{m}^{2}\right)+1.13 \times$ impaired fasting glycemia or diabetes (yes $=1 ;$ no $=0$ ) $+0.99 \times$ AST/ALT ratio- $0.013 \times$ platelet count $\left(\times 10^{9} / \mathrm{L}\right)-0.66 \times$ albumin $(\mathrm{g} / \mathrm{dL})$. 


\section{Statistical analysis}

Statistical analysis was performed using SAS 9.4 (SAS Institute Inc., Cary, NC, USA), and all figures were created using GraphPad Prism 7 for Windows (GraphPad Software, La Jolla, CA, USA). Normally distributed data were presented as number (\%) and mean $\pm \mathrm{SD}$, and non-normally distributed data were presented as the median with interquartile range. Categorical variables, ex-

Table 1. Baseline characteristics of patients with NAFLD

\begin{tabular}{lc}
\hline Clinical characteristics & Total (N=113) \\
\hline Age (yr) & $47.0[30.0 ; 61.0]^{*}$ \\
Sex & \\
Male & $58(51.3 \%)$ \\
Female & $55(48.7 \%)$ \\
Body weight (kg) & $76.0[67.8 ; 89.0]^{*}$ \\
Height (m) & $1.6 \pm 0.1$ \\
BMl (kg/m²) & $28.7[26.0 ; 31.2]^{*}$ \\
DM & $36(31.9 \%)$ \\
M2BPGi & $0.6[0.3 ; 1.1]^{*}$ \\
Steatosis & \\
1: 5-33\% & $45(39.8 \%)$ \\
2: 33-66\% & $38(33.6 \%)$ \\
3:>66\% & $30(26.5 \%)$ \\
Lobular inflammation & \\
0: none & $3(2.7 \%)$ \\
1: <2 foci per 200xfield (\%) & $63(55.8 \%)$ \\
2: 2-4 foci per 200x field (\%) & $42(37.2 \%)$ \\
3: >4 foci per 200x field (\%) & $5(4.4 \%)$ \\
Ballooning & \\
0: none & $26(23.0 \%)$ \\
1: mild, few & $39(34.5 \%)$ \\
2: moderate to marked, many & $48(42.5 \%)$ \\
Fibrosis & \\
0: none & $19(16.8 \%)$ \\
1a: mild, zone 3, perisinusoidal & $26(23.0 \%)$ \\
1b: moderate, zone 3, perisinusoidal & $6(5.3 \%)$ \\
1c: portal/periportal & $2(1.8 \%)$ \\
2: perisinusoidal and portal/periportal & $27(23.9 \%)$ \\
3: bridging fibrosis & $20(17.7 \%)$ \\
4: cirrhosis & $13(11.5 \%)$ \\
NAS & $4(4 ; 5)^{*}$ \\
1-4 & $57(50.4 \%)$ \\
5-8 & $56(49.6 \%)$ \\
\hline medan & \\
\hline
\end{tabular}

*median [25-75\% interquartile range].

Abbreviations: BMI, body mass index; DM, diabetes mellitus; M2BPGi, Mac2 binding protein glycosylation isomer; NAFLD, non-alcoholic fatty liver disease; NAS, NAFLD activity score. pressed as proportions, were compared using the Chi-square test. The diagnostic performances of all markers were expressed as diagnostic specificity, sensitivity, and area under the ROC curve (AUC). Delong's test [24] was performed to compare AUC values and C-statistics to evaluate the discriminative ability of the fibrosis markers. To compare liver fibrosis marker values among multiple groups according to liver fibrosis stage, we used the Kruskal-Wallis test and post-hoc comparison with the DwassSteel test for each paired comparison [25]. Spearman's rank correlation analysis was performed to determine the correlation between liver fibrosis marker level and liver fibrosis stage. To identify variables (M2BPGi, APRI, FIB-4, and NFS) associated with $\mathrm{F}>2$ or $\mathrm{F}>3$, we performed logistic regression analysis with continuous variables, and the results were expressed as odds ratio (OR) and $95 \%$ confidence interval $(\mathrm{Cl}) . P<0.05$ was considered statistically significant.

\section{RESULTS}

Patient characteristics are summarized in Table 1. Nineteen (16.8\%) patients had no fibrosis (F0), 34 (30.1\%) had F1, 27

Table 2. Clinical characteristics and laboratory data of patients with NAFLD according to liver fibrosis stage

\begin{tabular}{lccr}
\hline Clinical characteristics & Fibrosis $\leq 2(\mathrm{~N}=79)$ & Fibrosis $>2(\mathrm{~N}=34)$ & \multicolumn{1}{c}{$P$} \\
\hline Age $(\mathrm{yr})$ & $36.0(23.5 ; 50.5)$ & $61.0(54.0 ; 66.0)$ & $<0.001$ \\
BMI $\left(\mathrm{kg} / \mathrm{m}^{2}\right)$ & $29.1(26.2 ; 32.3)$ & $27.6(25.1 ; 30.1)$ & 0.077 \\
DM & & & 0.007 \\
$\quad$ No & $60(75.9 \%)$ & $17(50.0 \%)$ & \\
Yes & $19(24.1 \%)$ & $17(50.0 \%)$ & \\
AST ( $\mu$ kat/L) & $1.1(0.8 ; 1.4)$ & $1.3(0.8 ; 2.1)$ & 0.171 \\
ALT ( $\mu$ kat/L) & $1.7(1.3 ; 2.7)$ & $1.0(0.6 ; 1.8)$ & $<0.001$ \\
GGT ( $\mu$ kat/L) & $1.1(0.8 ; 1.8)$ & $1.6(1.1 ; 2.6)$ & 0.013 \\
Albumin $(\mathrm{g} / \mathrm{L})$ & $46.8 \pm 3.4$ & $43.2 \pm 4.6$ & $<0.001$ \\
Platelet $\left(\times 10^{9} / \mathrm{L}\right)$ & $249.0(210.5 ; 294.5)$ & $174.5(137.0 ; 228.0)$ & $<0.001$ \\
Total cholesterol (mmol/L) & $5.0(4.3 ; 5.7)$ & $4.2(3.7 ; 4.9)$ & 0.005 \\
M2BPGi (COI) & $0.5(0.3 ; 0.7)$ & $1.3(0.8 ; 2.2)$ & $<0.001$ \\
NAS & $4.0(4.0 ; 5.0)$ & $5.0(4.0 ; 5.0)$ & 0.314 \\
APRI & $0.7(0.5 ; 0.9)$ & $1.2(0.8 ; 1.6)$ & $<0.001$ \\
FIB-4 & $0.9(0.5 ; 1.5)$ & $3.9(2.5 ; 5.5)$ & $<0.001$ \\
NFS & $-2.9 \pm 1.4$ & $0.1 \pm 1.7$ & $<0.001$ \\
\hline AbbreV & &
\end{tabular}

Abbreviations: ALT, alanine aminotransferase; APRI, aspartate aminotransferaseto platelet ratio index; AST, aspartate aminotransferase; BMI, body mass index; COI, cut-off index; DM, diabetes mellitus; FIB-4, fibrosis index based on four factors; GGT, gamma-glutamyl transpeptidase; NAFLD, nonalcoholic fatty liver disease; NAS, NAFLD activity score; NFS, NAFLD fibrosis score; M2BPGi, Mac-2 binding protein glycosylation isomer. 
(23.9\%) had F2, 20 (17.7\%) had F3, and 13 (11.5\%) had cirrhosis (F4). The clinical and biochemical characteristics according to liver fibrosis stage ( $F \leq 2$ and $F>2$ ) are detailed in Table 2. The age of patients in the $F \leq 2$ group was lower than that of patients in the $\mathrm{F}>2$ group. Patients with DM were more frequently observed $(\mathrm{OR}=3.158,95 \% \mathrm{Cl}=1.353-7.369, P=0.007)$ in the $\mathrm{F} \leq 2$ group.

Serum M2BPGi levels and other marker values according to liver fibrosis stage are shown in Fig. 1. The AUC, cut-off value, sensitivity, and specificity of the liver fibrosis scores, including M2BPGi level, are given in Table 3. The diagnostic performances of M2BPGi, NFS, FIB-4, and APRI were the best for F>3, F>3, $F>2$, and $F>1$, respectively. Only the AUC of APRI for diagnosing $\mathrm{F}>0$ was not statistically significant (Table 3 ).

No significant differences were observed when the AUC values of M2BPGi and the other fibrosis markers were compared
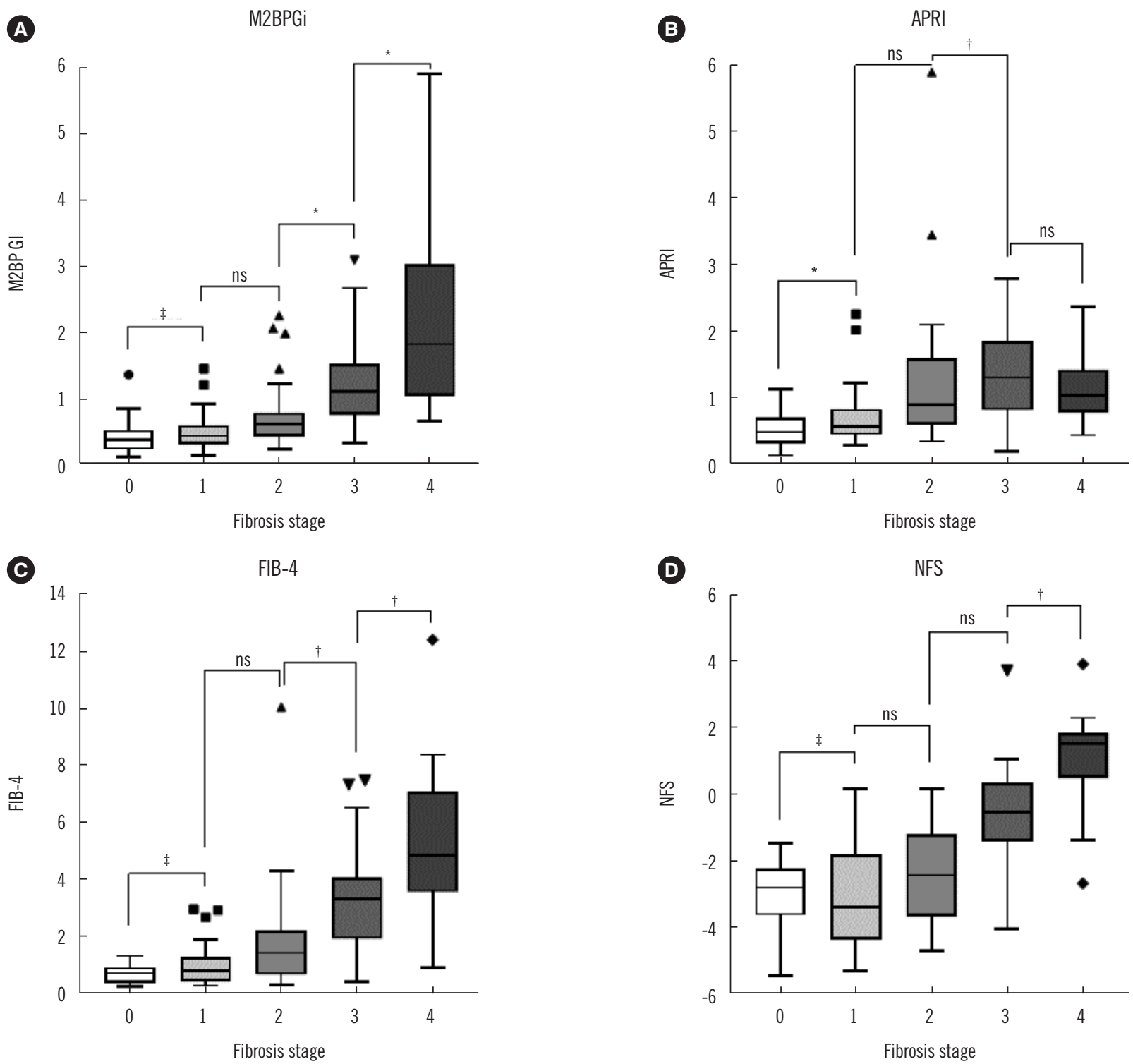

Fig. 1. Liver fibrosis marker values at each liver fibrosis stage in NAFLD patients. Spearman's rank correlation analysis showed that the serum M2BPGi level $(\rho=0.653, P<0.001)$ and FIB-4 $(\rho=0.689, P<0.001)$ increased with the liver fibrosis stage. There were moderate correlations between APRI $(\rho=0.515, P<0.001)$ and NFS $(\rho=0.628, P<0.001)$ and fibrosis stage, respectively. However, the median APRI score decreased in F4 than in F3, and median NFS decreased in F1 than in F0. (A) Serum M2BPGi level, (B) APRI, (C) FIB-4, and (D) NFS. The box height represents the interquartile range, and the line across each box represents the median. ${ }^{\star} P<0.05,{ }^{\dagger} P<0.01,{ }^{\ddagger} P<0.001$. Abbreviations: APRI, aspartate transaminase to platelet ratio index; FIB-4, fibrosis index based on four factors; M2BPGi, Mac-2 binding protein glycosylation isomer; NAFLD, non-alcoholic fatty liver disease; NFS, NAFLD fibrosis score; ns, not significant. 
Table 3. Diagnostic efficacy of serum M2BPGi and other serum markers for different liver fibrosis stages

\begin{tabular}{|c|c|c|c|c|c|}
\hline & AUC (95\% Cl) & $\begin{array}{l}\text { Cut-off } \\
\text { value }\end{array}$ & $\begin{array}{c}\text { Sensitivity } \\
(\%)\end{array}$ & $\begin{array}{l}\text { Specificity } \\
(\%)\end{array}$ & $P$ \\
\hline \multicolumn{6}{|l|}{ M2BPGi } \\
\hline $\mathrm{F}>0$ & $0.761(0.648-0.875)$ & 0.54 & 66.0 & 78.9 & 0.004 \\
\hline$F>1$ & $0.819(0.743-0.895)$ & 0.65 & 70.5 & 84.6 & 0.005 \\
\hline$F>2$ & $0.866(0.791-0.940)$ & 0.71 & 85.3 & 78.5 & $<0.001$ \\
\hline$F>3$ & $0.900(0.829-0.972)$ & 0.90 & 92.3 & 77.0 & $<0.001$ \\
\hline \multicolumn{6}{|l|}{ APRI } \\
\hline $\mathrm{F}>0$ & $0.778(0.676-0.880)$ & 0.76 & 61.7 & 89.5 & 0.109 \\
\hline$F>1$ & $0.793(0.708-0.877)$ & 0.76 & 77.0 & 75.0 & $<0.001$ \\
\hline$F>2$ & $0.758(0.661-0.855)$ & 0.76 & 88.2 & 62.0 & $<0.001$ \\
\hline$F>3$ & $0.641(0.497-0.786)$ & 0.76 & 84.6 & 52.0 & $<0.001$ \\
\hline \multicolumn{6}{|l|}{ FIB-4 } \\
\hline $\mathrm{F}>0$ & $0.783(0.694-0.871)$ & 1.39 & 56.4 & 100.0 & $<0.001$ \\
\hline$F>1$ & $0.852(0.781-0.922)$ & 1.23 & 78.7 & 84.6 & $<0.001$ \\
\hline$F>2$ & $0.918(0.857-0.980)$ & 1.70 & 91.2 & 79.7 & $<0.001$ \\
\hline$F>3$ & $0.894(0.801-0.986)$ & 3.30 & 84.6 & 87.0 & $<0.001$ \\
\hline \multicolumn{6}{|l|}{ NFS } \\
\hline$F>0$ & $0.687(0.586-0.788)$ & -1.83 & 54.3 & 89.5 & $<0.001$ \\
\hline$F>1$ & $0.820(0.743-0.898)$ & -1.62 & 68.9 & 90.4 & $<0.001$ \\
\hline$F>2$ & $0.920(0.862-0.977)$ & -1.04 & 79.4 & 93.7 & $<0.001$ \\
\hline$F>3$ & $0.926(0.841-1.000)$ & 0.25 & 84.6 & 94.0 & $<0.001$ \\
\hline
\end{tabular}

Abbreviations: AUC, area under the curve; APRI, aspartate aminotransferase to platelet ratio index; F, fibrosis; FIB-4, fibrosis index based on four factors; NAFLD, non-alcoholic fatty liver disease; NFS, NAFLD fibrosis score; M2BPGi, Mac-2 binding protein glycosylation isomer.

using DeLong's test, except for the difference between the AUC values of M2BPGi and APRI for diagnosing F>3 $(P<0.001)$. The univariate and multivariate logistic regression analyses and C-statistics results are shown in Table 4. Univariate logistic regression analyses showed that M2BPGi, APRI, FIB-4, and NFS were all associated with $F>2$ and $F>3$. Multivariate logistic regression analyses showed that M2BPGi was the only independent factor associated with $F>2$ and $F>3$. C-statistics showed that NFS was the best predictor for $F>2$ and $F>3$, with AUC values of 0.92 and 0.93 , respectively. When all the variables were included to construct a prediction model (model 1), the Cstatistics was 0.95. Combining M2BPGi and NFS as significant variables in the prediction model (model 2; following selection using a stepwise method) yielded a C-statistics of 0.94 for predicting $F>2$ and F $>3$ (Table 4).

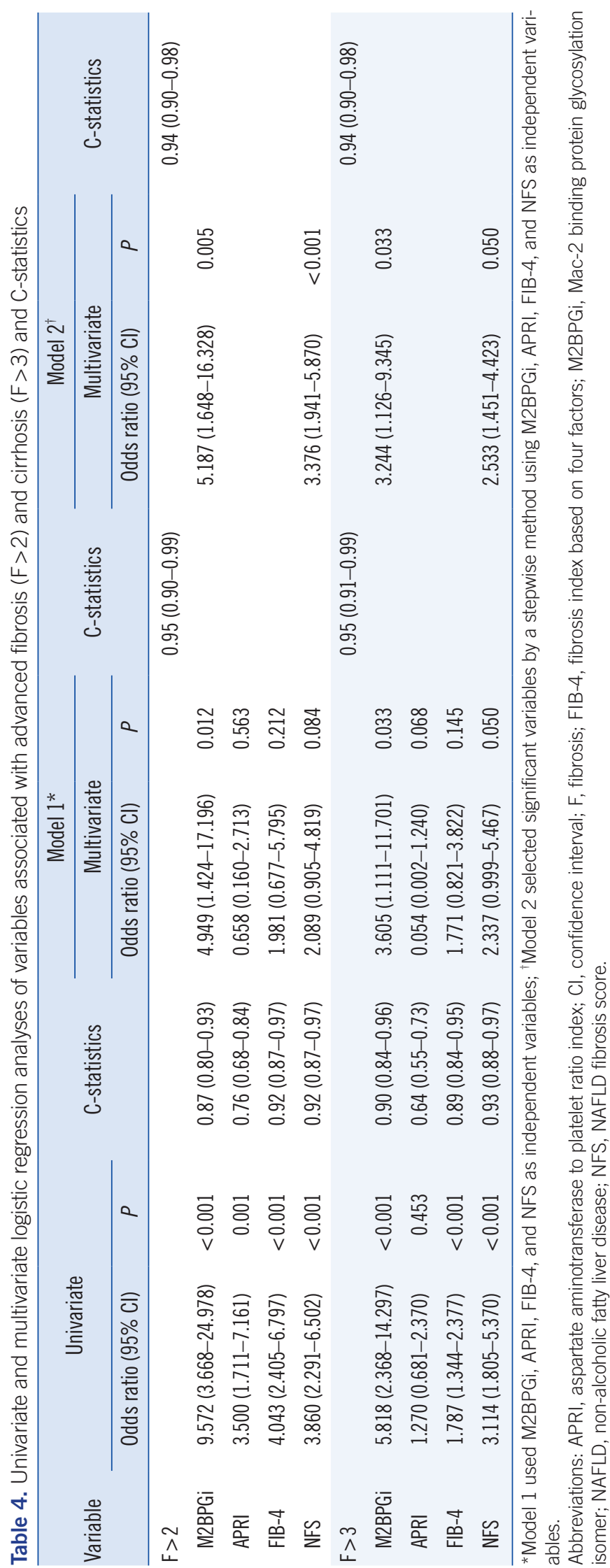




\section{DISCUSSION}

In our study, M2BPGi as well as FIB-4 and NFS showed moderate correlation with fibrosis stage and good diagnostic performance for advanced fibrosis or cirrhosis in NAFLD patients. Known liver fibrosis scoring systems have been introduced, including those based on biochemical factors, such as AST, ALT, albumin levels, and platelet count as well as those based on clinical factors, such as age, BMI, and the presence of impaired fasting glycemia or diabetes. In comparison to obtaining these scores, M2BPGi measurement in serum samples is relatively convenient and does not require further analysis.

Since serum M2BPGi was first introduced as a biomarker in Japan, it has been the subject of several studies [26]. In a study on 352 patients [8], the AUC of liver stiffness predicted by MRE for histologically advanced fibrosis ( $F>2$ ) was 0.910, and the optimal cut-off value was $4.07 \mathrm{kPa}$. M2BPGi was superior to FIB-4, NFS, and APRI in predicting severe stiffness over 4.07 $\mathrm{kPa}$. Another study on 325 patients showed that M2BPGi was a better predictor of $\mathrm{F}>2$ and $\mathrm{F}>3$ than APRI, FIB-4, and NFS [9].

The abovementioned studies considered M2BPGi to be the best predictor for advanced fibrosis $(F>2)$. However, our results showed that the performance of serum M2BPGi in diagnosing liver fibrosis was lower than that of FIB-4 and NFS in NAFLD patients. In addition, we did not observe significant differences between the diagnostic performances of the fibrosis scores. Based on $\mathrm{C}$-statistics, NFS was the best predictor for $\mathrm{F}>2$ and $\mathrm{F}>3$. Combining M2BPGi with NFS increased the $\mathrm{C}$-statistic values for predicting $\mathrm{F}>2$ and $\mathrm{F}>3$ by $1 \%$ and $2 \%$, respectively. We hypothesize that this is because our sample size was quite smaller than those of previous studies, resulting in a wide confidence interval range, which hindered accurate outcome prediction.

This study has several limitations. First, it was a single-center study with a smaller number of patients compared with other such studies conducted in Japan [4, 9]; this may be the reason for the low diagnostic efficacy of serum M2BPGi in this study than in previous studies. Second, histological examination was carried out by a single pathologist. Although inter-observer variation may have been avoided, interpretation bias may have occurred. Third, collected sera were stored at $-80^{\circ} \mathrm{C}$, and M2BPGi was not measured at the time of blood sampling; it was measured later but simultaneously for all samples. Contrarily, laboratory data of the other liver fibrosis markers were evaluated at the time of blood sampling. Differences in measurement times may have caused errors in the results and the low accuracy of serum M2BPGi-based fibrosis diagnosis in our study (median [range] of M2BPGi was 0.6 [0.13-5.90]]. In a Japanese study examining M2BPGi in NAFLD [27], the serum M2BPGi COI was 0.85 (0.22-11.32), slightly higher than the present data; however, another study showed different serum M2BPGi COls for nonHCC and HCC NAFLD (1.1 \pm 0.7 and 3.1 \pm 3.2 , respectively) [4]. Fourth, this study was retrospective in nature, and, as TE was not conducted for all patients, TE data could not be analyzed. However, European guidelines [28] and a meta-analysis [29] have reported that TE is more accurate than other serum fibrosis markers for predicting cirrhosis.

Despite its limitations, to the best of our knowledge, this is the first study to evaluate the serum M2BPGi level in NAFLD patients in Korea. Following the recent application of serum M2BPGi in clinical practice in Korea, there have been several reports on M2BPGi [10-13]. Two studies examined the diagnostic performance of serum M2BPGi for liver fibrosis screening during health checkups [11, 12]; one study examined the diagnostic efficacy of serum M2BPGi compared with those of other biomarkers in patients with chronic hepatitis B [10], and one study examined the diagnostic performance of serum M2BPGi and other biomarkers, including TE, in chronic liver disease [13]. These studies reported that, overall, serum M2BPGi is a good surrogate marker for predicting advanced fibrosis; however, various other studies have reported its superiority [11] as well as inferiority [10, 12, 13] compared with other serum fibrosis markers. In health checkup studies [11, 12], M2BPGi was measured in fresh serum samples, while, in other studies, residual samples stored at -70 or $-80^{\circ} \mathrm{C}$ were used to measure serum M2BPGi levels. Factors affecting a study may include liver disease etiology, number of patients, patient characteristics, and time of sampling and measurement.

In conclusion, our findings indicate that the serum M2BPGi level increased with liver fibrosis severity and could serve as a good marker for diagnosing advanced fibrosis and cirrhosis in NAFLD patients. Moreover, our results suggest that serum M2BPGi level combined with NFS can act as a better prediction model for advanced fibrosis and cirrhosis. A well-controlled, prospective study with a larger sample size should be conducted in the future to validate the diagnostic power of serum M2BPGi level and other markers for liver fibrosis.

\section{ACKNOWLEDGEMENTS}

Sysmax Company provided reagents and equipment for testing the samples in this study. Sysmax Company was not involved in study design, sample collection, or data analysis. 


\section{AUTHOR CONTRIBUTIONS}

Investigation: Jang SY, Han MH, Kim G, and Hur K; Resources: Park SY, Kweon YO, and Lee YR; Formal analysis: Lee WK; Funding acquisition: Jang SY; Writing-original draft: Jang SY; and Writing-review and editing: Tak WY.

\section{CONFLICTS OF INTEREST}

No potential conflicts of interest relevant to this article are reported.

\section{RESEARCH FUNDING}

This work was supported by Biomedical Research Institute grant, Kyungpook National University Hospital (2015).

\section{ORCID}

Se Young Jang https://orcid.org/0000-0001-9148-9670

Won Young Tak https://orcid.org/0000-0002-1914-5141

Soo Young Park https://orcid.org/0000-0002-4944-4396

Young-Oh Kweon

Yu Rim Lee

Gyeonghwa Kim

Keun Hur

Man-Hoon Han

Won Kee Lee

https://orcid.org/0000-0001-5708-7985

https://orcid.org/0000-0003-1916-1448

https://orcid.org/0000-0002-3795-4735

https://orcid.org/0000-0002-6944-2718

https://orcid.org/0000-0001-8856-553X

https://orcid.org/0000-0003-4217-5792

\section{REFERENCES}

1. Mak LY, Ko M, To E, Wong DK, Ma JH, Hui TL, et al. Serum Mac-2-binding protein glycosylation isomer and risk of hepatocellular carcinoma in entecavir-treated chronic hepatitis B patients. J Gastroenterol Hepatol 2019;34:1817-23.

2. Shinkai N, Nojima M, lio E, Matsunami K, Toyoda H, Murakami S, et al. High levels of serum Mac-2-binding protein glycosylation isomer (M2BPGi) predict the development of hepatocellular carcinoma in hepatitis $B$ patients treated with nucleot(s)ide analogues. J Gastroenterol 2018;53: 883-9.

3. Nagata H, Nakagawa M, Nishimura-Sakurai Y, Asano Y, Tsunoda T, Miyoshi $\mathrm{M}$, et al. Serial measurement of Wisteria floribunda agglutinin positive Mac-2-binding protein is useful for predicting liver fibrosis and the development of hepatocellular carcinoma in chronic hepatitis $\mathrm{C}$ patients treated with IFN-based and IFN-free therapy. Hepatol Int 2016;10:95664.

4. Kawanaka M, Tomiyama Y, Hyogo H, Koda M, Shima T, Tobita H, et al. Wisteria floribunda agglutinin-positive Mac-2 binding protein predicts the development of hepatocellular carcinoma in patients with non-alcoholic fatty liver disease. Hepatol Res 2018;48:521-8.

5. Nishikawa H, Enomoto H, Iwata Y, Hasegawa K, Nakano C, Takata R, et al. Clinical significance of serum Wisteria floribunda agglutinin positive Mac-2-binding protein level and high-sensitivity C-reactive protein concentration in autoimmune hepatitis. Hepatol Res 2016;46:613-21.

6. Umemura T, Joshita S, Sekiguchi T, Usami Y, Shibata S, Kimura T, et al. Serum Wisteria floribunda agglutinin-positive Mac-2-Binding protein level predicts liver fibrosis and prognosis in primary biliary cirrhosis. Am J Gastroenterol 2015;110:857-64.

7. Nishikawa H, Enomoto H, Iwata Y, Hasegawa K, Nakano C, Takata R, et al. Impact of serum Wisteria floribunda agglutinin positive Mac-2-binding protein and serum interferon-gamma-inducible protein-10 in primary biliary cirrhosis. Hepatol Res 2016;46:575-83.

8. Tamaki N, Higuchi M, Kurosaki M, Kirino S, Osawa L, Watakabe K, et al. Wisteria floribunda agglutinin-positive mac-2 binding protein as an age-independent fibrosis marker in nonalcoholic fatty liver disease. Sci Rep 2019;9:10109.

9. Abe M, Miyake T, Kuno A, Imai Y, Sawai Y, Hino K, et al. Association between Wisteria floribunda agglutinin-positive Mac-2 binding protein and the fibrosis stage of non-alcoholic fatty liver disease. J Gastroenterol 2015;50:776-84.

10. Jekarl DW, Choi H, Lee S, Kwon JH, Lee SW, Yu H, et al. Diagnosis of liver fibrosis with Wisteria floribunda agglutinin-positive Mac-2 binding protein (WFA-M2BP) among chronic hepatitis B patients. Ann Lab Med 2018;38:348-54.

11. Kim M, Jun DW, Park H, Kang BK, Sumida Y. Sequential combination of FIB-4 followed by M2BPGi enhanced diagnostic performance for advanced hepatic fibrosis in an average risk population. J Clin Med 2020; 9:1119.

12. Nah EH, Cho S, Kim S, Kim HS, Cho HI. Diagnostic performance of Mac2 binding protein glycosylation isomer (M2BPGi) in screening liver fibrosis in health checkups. J Clin Lab Anal 2020;34:e23316.

13. Moon HW, Park M, Hur M, Kim H, Choe WH, Yun YM. Usefulness of enhanced liver fibrosis, glycosylation isomer of Mac-2 binding protein, galectin-3, and soluble suppression of tumorigenicity 2 for assessing liver fibrosis in chronic liver diseases. Ann Lab Med 2018;38:331-7.

14. Younossi ZM, Blissett D, Blissett R, Henry L, Stepanova M, Younossi Y, et al. The economic and clinical burden of nonalcoholic fatty liver disease in the United States and Europe. Hepatology 2016;64:1577-86.

15. Younossi ZM, Koenig AB, Abdelatif D, Fazel Y, Henry L, Wymer M. Global epidemiology of nonalcoholic fatty liver disease-Meta-analytic assessment of prevalence, incidence, and outcomes. Hepatology 2016;64:7384.

16. Yang JD, Hainaut P, Gores GJ, Amadou A, Plymoth A, Roberts LR. A global view of hepatocellular carcinoma: trends, risk, prevention and management. Nat Rev Gastroenterol Hepatol 2019;16:589-604.

17. Regev A, Berho M, Jeffers LJ, Milikowski C, Molina EG, Pyrsopoulos NT, et al. Sampling error and intraobserver variation in liver biopsy in patients with chronic HCV infection. Am J Gastroenterol 2002;97:2614-8.

18. Kleiner DE, Brunt EM, Van Natta M, Behling C, Contos MJ, Cummings $\mathrm{OW}$, et al. Design and validation of a histological scoring system for nonalcoholic fatty liver disease. Hepatology 2005;41:1313-21.

19. Toshima T, Shirabe K, Ikegami T, Yoshizumi T, Kuno A, Togayachi A, et al. A novel serum marker, glycosylated Wisteria floribunda agglutininpositive Mac-2 binding protein (WFA(+)-M2BP), for assessing liver fibrosis. J Gastroenterol 2015;50:76-84.

20. Yamasaki K, Tateyama M, Abiru S, Komori A, Nagaoka S, Saeki A, et al. Elevated serum levels of Wisteria floribunda agglutinin-positive human Mac-2 binding protein predict the development of hepatocellular carcinoma in hepatitis C patients. Hepatology 2014;60:1563-70.

21. Wai CT, Greenson JK, Fontana RJ, Kalbfleisch JD, Marrero JA, Conjeevaram $\mathrm{HS}$, et al. A simple noninvasive index can predict both significant 
fibrosis and cirrhosis in patients with chronic hepatitis C. Hepatology 2003;38:518-26.

22. Sterling RK, Lissen E, Clumeck N, Sola R, Correa MC, Montaner J, et al. Development of a simple noninvasive index to predict significant fibrosis in patients with HIV/HCV coinfection. Hepatology 2006;43:1317-25.

23. Angulo P, Hui JM, Marchesini G, Bugianesi E, George J, Farrell GC, et al. The NAFLD fibrosis score: a noninvasive system that identifies liver fibrosis in patients with NAFLD. Hepatology 2007;45:846-54.

24. DeLong ER, DeLong DM, Clarke-Pearson DL. Comparing the areas under two or more correlated receiver operating characteristic curves: a nonparametric approach. Biometrics 1988;44:837-45.

25. Douglas CE and Michael FA. On distribution-free multiple comparisons in the one-way analysis of variance. Communications in Statistics - Theory and Methods 1991;20:127-39.

26. Tamaki N, Kurosaki M, Loomba R, Izumi N. Clinical utility of Mac-2 Bind- ing Protein Glycosylation Isomer in chronic liver diseases. Ann Lab Med 2021:41:16-24.

27. Atsukawa M, Tsubota A, Okubo T, Arai T, Nakagawa A, Itokawa N, et al. Serum Wisteria floribunda agglutinin-positive Mac-2 binding protein more reliably distinguishes liver fibrosis stages in non-alcoholic fatty liver disease than serum Mac-2 binding protein. Hepatol Res 2018;48:424-32.

28. European Association for Study of Liver; Asociacion Latinoamericana para el Estudio del Higado. EASL-ALEH Clinical Practice Guidelines: non-invasive tests for evaluation of liver disease severity and prognosis. J Hepatol 2015;63:237-64.

29. Xiao G, Zhu S, Xiao X, Yan L, Yang J, Wu G. Comparison of laboratory tests, ultrasound, or magnetic resonance elastography to detect fibrosis in patients with nonalcoholic fatty liver disease: a meta-analysis. Hepatology 2017;66:1486-501. 